\title{
Examine the Perception of Contemporary Midwives and Pre Service Midwives About the Practice of Traditional Birth Attendants in Kwasmintim District
}

\author{
Hannah Yawson (PhD Student) \\ Principal, Twifo Praso Nurses and Midwifery Training College
}

\begin{abstract}
The paper focused on examining the perception of contemporary midwives and pre-service midwives about the practice of Traditional Birth Attendants (TBAs) in Kwesimintsim District. It is believed that the success of the programme of Traditional Birth Attendants practice is dependent strongly on the cooperation and collaboration with midwives. Therefore, determining the perception of this key stakeholders (contemporary midwives and preservice midwives) is fundamental to the quality maternal health care system: this is the motivation for this paper. The paper followed qualitative analytical approach with exploratory design. It emerged that despite the existing programme for TBAs practice, majority of the midwives and pre-service midwives perceived their practice as dangerous. It was also found The TBAs were perceived as though diligent but stereotyped, conservative and unwilling to sacrifice traditions and cultural for modern practices. It is recommended that the skilled birth attendants (Midwives and pre service midwives) should be engaged in the TBA programme for effective partnership. It is further recommended that community service with TBAs should be mandatory as part of the pre service programme for midwives.
\end{abstract}

Keywords: Traditional Birth Attendants, Skilled Birth Attendants, Midwives, and Pre-service Midwives

DOI: $10.7176 / \mathrm{JHMN} / 94-04$

Publication date:October $31^{\text {st }} 2021$

\section{Introduction}

The Global health issues that have become predominate over the years is the child mortality. This global crisis has drawn the attention of government, NGO, policy makers, health practitioners as well as researchers to investigate the causes of those issues. The prevention of child mortality continues to be on the radar of global health forum. As such the current Sustainable Development Goals, in its goal three (3) proposes to among other health related problems end child mortality by 2030 (Bryce, Black \& Victora, 2013; Yawson, 2019). This agenda is very relevant due to the deteriorating infant mortality. According to WHO, it is estimated that each year around 2.7 million newborns die in the first month of lives, worldwide and an estimated 303,000 women die from complications related to pregnancy or childbirth (Bayou, 2014). This number of death resulting from pregnancy and child birth is not only alarming but detrimental to upward scaling of global development (Yawson, 2019).

Global health experts and international organisations are continuously reviving their strategies ad programme to defeat this frightening health canker. In fact, section 3.2 of the Sustainable Development Goal (SDG) proposes to have by 2030, end preventable death of newborns and children under 5 years of age, with all countries aiming to reduce neonatal mortality to at least as low as 12 per 1,000 live births and under 5 mortalities to a least a low as 25 per 1,000 live births (World Health Organisation, WHO, 2019). To achieve this important feat, pregnant women of all sort including those in the rural and deprived communities should be treated equally with reasonable access to quality health care.

Despite the global efforts and country level governmental interventions to reduce maternal and infant mortality, countries within low and middle income zones continue to report disturbing significant mortality rates, due to among others poor access to quality of professional health care. One of the approaches for addressing the menace is to regularise the practice of traditional birth attendants (TBAs). TBAs have historically been considered not only as the major caregivers but also respectable birth attendants for women during childbirth in Africa (AbdulMumin, 2016; Aborigo, Allotey \& Reidpath, 2015). This is no exception in Ghana. In Ghana, expectant mothers continue to either give birth themselves at home or with TBAs (Aziato, \& Omenyo, 2018).

According to the World Health Organisation (WHO), a traditional birth attendant (TBA) is a person who assists the mother during childbirth and who initially acquired her skills by delivering babies herself or by working with other TBAs. In addition to attending deliveries, TBAs help with initiating breastfeeding; providing health education on sexually transmitted illnesses (STIs), reproductive health and nutrition; visiting mothers during and shortly following delivery to check for and educate them on the associated danger signs; and accompanying referrals to the health facilities for complicated deliveries (Byrne \& Morgan 2011). The role of TBAs varies across cultures and times, but, even today, they attend the majority of deliveries in rural areas of developing countries. There is no doubt that they have a significant role when it comes to cultural competence, consolation, empathy, and psychosocial support at birth, all of which are important benefits for the mother and also for the new-born 
child (Sumankuuro, 2014).

Although the enhancing contemporary midwifery education seeks to shift the recognition of TBAs to skill birth attendants (SBA), the unfulfilled hopes of reducing child mortality and safe delivery have called the world to rethink through their health policies. One of the options was to formally call the services of TBAs again and regularise the practice (Ana, 2011; Yawson, 2019). This shift seeks to use the services of TBAs to complement efforts aimed at improving maternal and perinatal health in low and middle-income countries (Ana, 2011). The effectiveness of this partnership and integration requires a strong collaboration between the TBAs and the SBAs (Midwives and pre service midwives). Unfortunately the integration continuous to receive backlash and fierce debate among health professionals worldwide (Aziato, \& Omenyo, 2018; Dzakpasu, Soremekun, Manu, Asbroek, Tawiah , Hurt , Fenty , Owusu-Agyei , 2012; Harrison, 2011; Sarelin, 2014). Meta-analysis conducted by Yawson (2019) revealed mixed results in the literature (Amjad et al, 2014; Ebuehi \& Akintujoye, 2012; Essendi, 2015; Kaingu, Oduma, \& Kanui, 2011; Nelms \& Gorski, 2006; Oshonwoh Ferdinand, Nwakwuo Geoffrey \& Ekiyor Christopher, 2014; Pfeiffer \& Mwaipopo, 2013; Sarmento, 2014; Selepe \& Thomas, 2000; Titaley et al, 2012; Vyagusa, Mubyazi, \& Masatu, 2013).

Notwithstanding the controversies about the integration of the TBAs, the role of TBAs will not end any time soon. Therefore, it is critical to contribute to improving their role rather than crying foul of their intervention and programme of integration. One means of improving the relevance of TBAs practice is to ensure effective collaboration with the fundamental partners-skilled birth attendants (SBAs). This is especially fundamental in Ghana as it has been observed from literature that Ghanaian women have traditionally preferred to deliver at home due to the fact that it is cheaper and easier as women who deliver at home mostly receive social support from their extended families and do not have to pay much for the delivery service (Crissman, Engmann, Adanu, Nimako, Crespo \& Moyer, 2013). It is against this that this paper seeks to explore the perception of midwives and pre service midwives about the practice of the TBAs in Ghana using data from Kwasmintim District.

\section{Literature Review}

This paper follows theory of culture care diversity and universality. The theory of Culture Care Diversity and Universality (TCCDU) is the creative outcome of independent thinking (Alligood, 2013). The roots of the theory reflect the theorist's early and current nursing practice and draws upon the theorist's experiences and creative thinking relevant to nursing and health fields. It has been independently developed and soundly constructed as a highly relevant theory to discover the care and health needs of diverse cultures in hospitals, clinics, community settings, and study of many cultures worldwide. The theory has become a major caring theory with a unique emphasis on nursing as a means to know and help cultures. Culturally based care factors are recognised as major influences upon human expressions related to health, illness, wellbeing, or to face death and disabilities. The theory has become meaningful as a guide to nurses' thinking, practices, and research. This process of envisioning and conceptualising care is the essence of nursing. The theorist postulates that human care is what makes people human, gives dignity to humans, and inspires people to get well and to help others and further predicts there can be no curing without caring, but caring can exist without curing (Bonder \& Martin, 2013; Mesoudi, 2011).

The theory focused on culture care as an interrelated phenomenon and crucial to help nurses discover and identify new ways to understand and advance nursing, healing, and health care. Leininger holds that care needs to become meaningful, explicit, and beneficial; it needs to be conceptualised showing the interrelationships of care to culture and to different cultures - the transcultural nursing focus. Care is a powerful and dynamic force to understand the totality of human behavior in health and illness. Action modes related to care that are culturally based and maintained beneficial health outcomes are needed. Care needs to be understood and actualised in diverse and specific cultural contexts.

A critical analysis of the theory shows that the culture care theory addresses the contentions of culture diversity. Applying these assumptions to the current study, one could argue that cultural values play significant role in the lives of individual including the choice of healthcare. Therefore a particular practice may be irrelevant in one area but may be highly embraced in another area. Given this assertion, members of a given community may still attach to TBAs services despite the availability of midwives or health centers due to socio-cultural and traditional reasons. Similarly, how the agent of change perceive their culture, treat them and care for them may also affect their decisions. Therefore the perception and attitude of the skilled birth attendants towards the community, its customs and tradition as well upholding their dignity as humans would influence the appetite for their services (Bonder \& Martin, 2013; Mesoudi, 2011). On the contrary where the SBAs do not envision and conceptual recognition and care as essence of nursing, then they would expect the reciprocal.

This suggests that it is not enough to presume that because of the current trend in midwifery education and practice in terms of the number, the society would embrace them and abandon their own folks. It would be quite expensive to society to make such shift. To sacrifice this rather perceived expensive practice for the main stream health care, they should be able to predict reasonably that they would be well cared and cater for. It is thus important to assess the perception and attitude of these SBAs towards the TBAs who uphold the traditions and 
preserve the culture of the community. Leininger holds that culture is the broadest, most comprehensive, holistic and universal feature of human beings and care is predicted to be embedded in culture (Riegel, Jaarsma, \& Strömberg, 2012). Both need to be understood to discover clients' care needs. Caring is held as the action mode to help people of diverse cultures while care is the phenomenon to be understood and to guide actions and decisions. Culture and care together are predicted to be powerful theoretical constructs essential to human health, wellbeing, and survival. In-depth knowledge of the specific culture care values, beliefs, and lifeway's of human beings within life's experiences is held as important to unlock a wealth of new knowledge for nursing and health practices. Thus, to wane the TBAs practice in the future would require SBAs to accept them now, learn their practices and polish them professionally so as to gradually win the culture over as well as the trust of the expectant mothers.

The theory of culture care also assumes care repatterning and-or restructuring which is referred to those assistive, supportive, facilitative, or enabling professional actions and mutual decisions that would help people to reorder, change, modify or restructure their lifeways and institutions for better (or beneficial) health care patterns, practices or outcomes (Papesh, 2013; Sayegh, \& Knight, 2013). This suggests that the professionals such as the midwives and pre-service nurses should consider a change in their attitude, modify their life style and practice so as to wining the caring for the community they intend to serve. This would require positive perception rather than negative perception about the traditions of the people. It is this good posture of the professionals that could change the people. Thus, the professional should undertake at least some changes first to suit the people, then he/she may be able to drag the people to accept his/her traditions (Agyemang, 2011). Therefore assessing the perceptions of these professions would provide empirical evidences to support or disaffirm this theory.

Besides the theoretical basis for assessing the perception of skilled birth attendants about TBAs' practice, some relevant empirical considerations provide direction for the investigation. Most of the existing empirical studies on the practice of TBAs have focused on the relevance and role of TBAs (Adatara, Afaya,Baku, Salia, \& Asempah, 2018; Ebuehi \& Akintujoye, 2012; Sarmento, 2014; Shaikh, Khan, Maab \& Amjad, 2014; Titaley, Dibley \& Roberts, 2012; Yawson, 2019). In spite of the fact that trained birth attendants at delivery are important for preventing both maternal and newborn deaths, Titaley, Dibley and Roberts (2012) realized that in West Java, one of the provinces on Java Island, Indonesia, many women still delivered at home and without the assistance of trained birth attendants. Titaley et al (2012) thus conducted a study aimed to explore the perspectives of community members and health workers about the use of delivery care services in six villages of West Java Province. A qualitative study approach using focus group discussions (FGDs) and in-depth interviews was conducted in six villages of three districts in West Java Province from within an interval of five month. Twenty FGDs and 165 indepth interviews were conducted involving a total of 295 participants representing mothers, fathers, health care providers, traditional birth attendants and community leaders. The FGD and in-depth interview guidelines included reasons for using a trained or a traditional birth attendant and reasons for having a home or an institutional delivery. The result revealed that the use of traditional birth attendants and home delivery were preferable for some community members despite the availability of the village midwife in the village. The limited availability of health care providers was reported by residents in remote areas. In these settings the village midwife, who was sometimes the only health care provider, frequently travelled out of the village. The community perceived the role of both village midwives and traditional birth attendants as essential for providing maternal and health care services.

It could be deduced from the study of Titaley et al (2012) that the relevance of the TBAs is as a result of the irregularities in the camp of the trained delivery attendants. Some of these irregularities include the frequent travels of the various midwives who reside in the villages. Accessibility and location of health centers contribute to the relevance of the TBAs in the West Java provinces of Indonesia according to the study of Titaley et al (2012). Although the study of Titaley et al (2012) has demonstrated the relevance of TBAs and the community acceptance, the safety of their practice requires collaboration from the SBAs. What is the perception of these SBAs about these TBAs' practice and to what extent are SBAs ready to accept these TBAs? These are important empirical questions which were not considered by Titaley et al (2012) and primary to the current paper. Although investigating the roles and relevance of TBAs are important to determine their contribution to the maternal healthcare system, to reduce maternal mortality goes beyond assessing the roles and relevance. It is fundamental to assess the readiness of SBAs to accept referral cases from TBAs and effective collaboration to handle complications as this precursor to reducing the maternal mortality.

Another stream of studies also focus on the knowledge, attitude and practice of TBAs (Amjad et al, 2014; Asweto, Aluoch, Obonyo, \& Ouma, 2014; Vyagusa, Mubyazi \& Masatu, 2013). These studies have contributed to building policies to support maternal health care delivery. For instance, Vyagusa et al (2013) observed that majority of the TBAs have low level of knowledge on delivery complications and also signs of pregnancy complications. Moreover, it was observed that the instrument or materials used for helping in child delivery were either below standard or unhygienic. These findings and conclusions provide evidence that collaboration with SBAs is critical for effectiveness of TBAs practice in supporting the maternal health care system. Unfortunately, the evidence about the perception and extent of readiness of SBAs to collaborate is still in its infancy with relative empirical based literature. This paper seeks to contribute to the literature by exploring the perception of SBAs 
about the practice of TBAs in Kwesimitim District in Ghana.

\subsection{Research Methodology}

This section of the paper describes the approaches and methods employed to conduct the study. The methods are presented based on the focus of the paper. Relevant justifications are provided in each thematic area of the methodology.

\subsection{Research Approach}

The paper employs qualitative approach. As indicated by Weinriech (2006), qualitative approach is appropriate when a study seeks to give priority to target audiences, their culture or situation and involves direct interaction with respondents. According to Creswell the appropriateness of the approach depend partly on the data requirements, data collection techniques and mode of analysis (Creswell, 2009). Therefore as non-numerical based study where the views, perceptions and intentions of the participants are central to the data collection and method of analysis, qualitative approach is suitable. The data needs are non-numerical where direct interactions with the respondent are the main source of data for this paper. This research approach is applied by gathering non-numeric data from the Skilled Birth Attendants (midwives and pre-service midwives).

\subsection{Research Design}

The paper uses qualitative explorative design. Exploratory design involves requires the search for individuals with adequate knowledge about the subject under investigation, solicit their views, opinions, beliefs and perceptions of the said subject. It also assists the researcher to formulate and understand the problems and clarify concepts. This design is useful in this paper because it would provide adequate insights and understanding of the subject and enhance discovery of new ideas (Yin 2009). The author explained further that the exploratory design helps the research to explore situations in which the intervention being evaluated has no clear or single set outcomes. It is also believed that the exploratory design is appropriate for qualitative study because it requires a design that supports non numerical data collection.

\subsection{Study Population and Sampling Methods}

The Skilled Birth Attendants (SBAs) from the Kwesimintsim in western region are the main participants of the study and therefore forms the target population. The target groups are those who have adequate knowledge about the subject. The exact population is not readily available, however, the study uses sample size of 35 . This comprises 15 midwives and 20 pre service midwives. Convenience sampling technique is applied to select the sample size of 35 .

\subsection{Data Collection and Analysis}

The paper employed interview as the mode of data collection. The interview is facilitated by interview guide which contained questions that inquired about the perceptions of SBAs on the practices TBAs in maternal health care in the areas. The interviews were done face-to-face with participants at their various homes and at their convenient time which lasted between 15 to 30 minutes. The interviews were recorded with the permission of participants. Subsequent to the data collection, the paper employed content and thematic analysis to analyse the data collected. The analysis is conducted manually. These analyses involve matching the data generated through the interview and attributing or relating consequences to antecedents (Polit \& Beck, 2004). The study first organises the field data, merge the field notes with tape-recorded data for the purpose of obtaining holistic sense of the data (De Vos, Strydom, Fouche, 2009). The data are coded for the purpose of generating relevant categories. The categories are generated according to their theoretical significance and importance to achieving the purpose of the paper.

Through the thematic analysis, data reduction is conducted without sacrificing the meaning expressed directly by the interviewees or the participants (Gillham, 2007; Queku, 2016). The study reduces the various categories into set of relevant themes (Creswell, 2009). The themes provide the basis for the data analysis and interpretations. Thus, the data interpretations are based on the themes so as to obtain deeper meaning and significance of the field data collected (Queku, 2016). The findings from the data analysis are considered as theoretical statement responding to the research questions formulated to address the corresponding specific objective. These findings are validated by samples or examples of direct quotes from the participants. The empirical and theoretical discussions of the results are presented based on these specific objectives. Thus, the outcomes of the analysis are situated in the literature by both theoretical and empirical comparison. Relevant implications noted and conclusions drawn.

\subsection{Results and Discussions}

This section of the paper discusses the results from the qualitative analysis. Even though the some of the SBAs expressed the important roles of TBAs services in the health care, majority of the midwives and pre-service 
midwives perceived their practice as dangerous. The perception of the SBAs (midwives and pre-service midwives) was developed from two main key sub-themes, namely, relationships with SBAs and scope of practices.

\subsection{Theme one: Skilled Birth Attendants Relationship with Traditional Birth Attendants}

Although some of the midwives and pre-services midwives believed that the practices of trained TBAs have contributed greatly to reducing maternal mortality, majority perceived them contrary. The findings in the study revealed that those midwives and pre-service midwives who had lived in rural areas and practiced in less endowed communities really appreciated the services of the TBAs and have had good relationship with the TBAs. One participant stated:

"I sometimes make time to visit some of the TBAs in this community, sit and chat with. I do spend significant time talking about their practices..........learn from them and also advise them. THEY ARE QUITE APPRECIATIVE" (MW\#3)

"I come from a community where TBA practice is common. I learnt under my grandmother and mother when I was a kid.................I developed the interest in midwifery from my family..... my mother used to diagnose some expectant mothers, identify a complication and specifically ask the mother to run some checks at hospital. Some of these women come back and confirm such complications (MW \#19)

Moreover, some of the interviewed SBAs believed that if one would like to be successful in the health profession and support the community, then she/he should build a cordial relationship with these TBAs. One respondent explained:

"Despite the continuous increase in the number of midwives and health centres, without the TBAs complement our efforts, it would be difficult to achieve the goal of the lowest mortality rate possible............................the expectant mothers have faith in these TBAs. We can get the mothers to trust us if they see that we trust their community members (TBAs)................” (MW\#2)

"The areas this health facility is to cover is too large to be managed...........Some of these TBAs are helping. I envisage deepening in this partnership program in the future. In fact we cannot overrun the TBAs" (PSM \# 15)

Notwithstanding these remarks, the findings indicated that majority of the SBAs have negative perception about the TBAs practice and this has strained their relationship. This study found that the SBAs perceived that the TBAs are sometimes over confidence and do not stick to direction and advice. The TBAs were perceived as though diligent but stereotyped, conservative and unwilling to sacrifice traditions and cultural for modern practices. Majority of the SBAs echoed that these TBAs wait at the last minutes before sending the complicated cases to us. Sometimes it becomes too late to save either the mother or the baby or both.

"I have lost a number of babies and cannot afford to lose another, I can't tolerate these old folks. Sometimes are stories are wield: THE SPIRIT TOLD ME THIS, THE gods SAID THAT..........people are dying. We can't continue to put up with them. They need to be held accountable" (MW\#1)

Many other midwives and pre-services nurses expressed similar sentiments like that of MW\#1.

"How can we as a nation achieve the target for maternal mortality rate when we gamble with the lives of the expectant mothers and babies. If there is no problem trust the service of the old women (traditional birth attendants) but where it looks complicated call the midwife" (MW\#4)

"I am told they have been trained to identify complication signs .just few days training how could they learn and adapt to these knowledge? Some of us have spent years but we are still not conversant..........THEIR SERVICES ARE RISKY, IN FACT WE ARE RISKING LIVES" (PSMW\#7)

"These TBAs would not give up some of their cultural-oriented practices....................the TBAs believe these practices are sustaining them and therefore cannot give them up.............I stopped collaborating with them when I saw that they were not ready to give up the ill-practices ." (MW \#9)

"My brother has been following the every midwife that comes here. He can now give injections and circumcision......... want to do some. If you people can teach me I would come to your meeting" (TBA \#10)untrained.

Most of the midwives expressed that some of the TBAs see them as competitors. Some revealed how they encountered severe resistance from some of the TBAs in their communities when they were deployed. Due to age disparities these TBAs are not often ready to accept directives from some of us.

"These TBAs see us as rivals; ............even if not better than us. They think the only difference is that we are paid but they not" (MW\#5)

"I think we cannot achieve our goal of reducing maternal mortality if we allow this so called partnership to exist..........................individual midwives should resist them.....

" (MW \# 25)

\subsection{Awareness and Scope of Traditional Birth Attendants Practices}

Absence of effective communication to midwives and pre-service midwives about the roles, definitions of scope of practice and partnership programme of TBAs to the health care system make most of the SBAs perceive the practice of the TBAs illegal. It was evident that lack of awareness among some of the SBAs about the opportunities 
given to the TBAs to practice child delivery has affected the perceptions of these SBAs. The study found that some of the SBAs in the study area were not aware of the formal partnership between midwives and traditional birth attendants as well as the scope of practice of these TBAs. For instance, some pre-service midwives were not aware that some TBAs have even been certified through training to conduct child delivery.

“........I don't know whether I have forgotten. The news is new to me. When was this programme instituted? May be because I am in school, I don't know what is happening out there" (PSMW\#5)

“.............I don't know whether it is true but still I think this partnership can't be possible. It would make our work very difficult...........I can't accept some of these people as my partner. Most of them are too old and illiterate what specifically can they do to assist me" (PSMW\#9)

" .............therefore I have always been against this programme. The duration to me was not enough to certify them to practice...........they are mostly aged and illiterate how could they internalise these skills within this short period?" (MW\#14)

"I have been hearing complains about ill-treatment of some of these expectant mothers by these TBAs. I have not seen these personally but our midwives have been complaining a lot about them....................... think their practice should be stopped ................ w would not accept them at my centre when I am posted....................................." (PSM \# 13)

"............... In my opinion, if we do not chase them now, we would not be able to chase them again as they would use their culture and the community members to resist us.........I cannot collaborate with them..................." (MW \# 23).

Some of the midwives perceived that some of the TBAs do not really know the actual permissive scope of their practice. Even those who could describe clearly their scope they hardly follow these due to their tradition. Other SBAs revealed because the TBAs do not abide by their scope and even conduct delivery on mothers who had been referred to deliver in a hospital due to cultural beliefs and traditions. It was evident that lack of awareness among some of the SBAs about the opportunities given to the TBAs to practice child delivery has affected the perceptions of these SBAs. Some even considered their practice as illegal. Majority of the midwives and preservice midwives who were aware perceived their practice as dangerous. Only the midwives and pre-service midwives who had lived in rural areas or practiced in less endowed communities really appreciated the services of the TBAs and have had good relationship with them. These relatively few believed that if one would like to be successful in the health profession and support the community, then she/he should build a cordial relationship with these TBAs.

This study found that the SBAs perceived the TBAs as over confidence and do not stick to direction and advice. They also perceived the TBAs as stereotyped, conservative and unwilling to sacrifice traditions and cultural for modern practices. Most of the midwives expressed that some of the TBAs believed they are their competitors. Some revealed how they encountered severe resistance from some of the TBAs in their communities when they were deployed. The negative perception and poor relationship between the midwives and the pre-service midwives about the TBAs revealed in this study provides empirical evidences to affirm the theory of culture care as discussed in chapter two (Ball, \& Olmedo, 2013). The findings also articulate the Leininger theory which argues that nurses in focusing on caring for humans should not look up to only their own culture but should also show the interrelationships of care to culture and to different cultures - the transcultural nursing focus (Riegel, Jaarsma, \& Strömberg, 2012). These tenets have been echoed in the findings in this present study. Empirically, Ameyaw (2011) also found negative perception of the practising midwives and pre-service midwives about the practice of the TBAs.

It can be concluded that the midwives and the pre-service midwives perceived the TBAs services as dangerous, risky and unacceptable. With these perceptions, the programme co-ordinators and health administrators should build platform where these two groups can actively interact to minimise the differences and draw goal congruent. The health institutions may also articulate management of this relationship through designing their courses to include knowledge of traditional practices.

\subsection{Summary and Conclusions}

The paper focused on exploring the perception of skilled birth attendants about the practice of traditional birth attendants in Kwesimintsim District. The paper operationalises the purpose into two main themes specific. The first part of the study focused on the relationship existing between skilled birth attendants and traditional birth attendants. The second and the final part looked at the awareness and Scope of Traditional Birth Attendants Practices.

Regarding the perceived relationship, it was found that although some of the midwives and pre-services midwives believed that the practices of trained TBAs have contributed greatly to reducing maternal mortality, majority perceived them contrary. The findings in the study revealed that those midwives and pre-service midwives who had lived in rural areas and practiced in less endowed communities really appreciated the services of the TBAs and have had good relationship with the TBAs. These relatively few believed that if one would like to be 
successful in the health profession and support the community, then she/he should build a cordial relationship with these TBAs. Notwithstanding these remarks, the findings indicated that majority of the SBAs have negative perception about the TBAs practice and this has strained their relationship. This study found that the SBAs perceived that the TBAs are sometimes over confidence and do not stick to direction and advice. The TBAs were perceived as though diligent but stereotyped, conservative and unwilling to sacrifice traditions and cultural for modern practices. Majority of the SBAs echoed that these TBAs wait at the last minutes before sending the complicated cases to us. Sometimes it becomes too late to save either the mother or the baby or both. Most of the midwives expressed that some of the TBAs see them as competitors. Some revealed how they encountered severe resistance from some of the TBAs in their communities when they were deployed. Due to age disparities these TBAs are not often ready to accept directives from some of us. It was concluded that midwives and pre-service midwives perceived the traditional birth attendants practice as dangerous.

Referencing the awareness and scope of TBAs' pracrice, it was found that the absence of effective communication to midwives and pre-service midwives about the roles, definitions of scope of practice and partnership programme of TBAs to the health care system make most of the SBAs perceive the practice of the TBAs illegal. It was evident that lack of awareness among some of the SBAs about the opportunities given to the TBAs to practice child delivery has affected the perceptions of these SBAs. The study found that some of the SBAs in the study area were not aware of the formal partnership between midwives and traditional birth attendants as well as the scope of practice of these TBAs. For instance, some pre-service midwives were not aware that some TBAs have even been certified through training to conduct child delivery. Some of the midwives perceived that some of the TBAs do not really know the actual permissive scope of their practice. Even those who could describe clearly their scope they hardly follow these due to their tradition. It was also concluded that midwives and preservice midwives perceived the traditional birth attendants as over confidence, stereotyped, conservative and unwilling to sacrifice traditions and cultural for modern practices.

\subsection{Recommendations}

Following the findings emanating from this paper coupled with the relevant conclusions reached, the paper presents the following recommendations to support policy SBAs and TBAs effective partnership. The findings showed ill-perception among the midwives and pre service about the practice of TBAs, therefore, Ministry of Health is advised to strengthening the partnership program and co-ordination between the traditional birth attendants and the midwives in the main health care system so as to build health relationship. This would make it easier for these TBAs to make referrals and some cases accompany their patients to health centers. Policy makers are advised to project a long term goal that would give birth to a midwifery program that would allow the midwives to be integrated as part of the communities they serve. This may be in a form of assisting in home delivery rather than taking the expectant mothers to the health centers. Eventually, the system may weed the TBAs practice completely. The findings revealed that some of the midwives and pre service midwives are completely at lost to the TBA programme and this might have informed their ill-feelings about the TBAs, it is therefore recommended that awareness creation should be intensified in various health centres and facilities as well as health training institution. The health training institutions such as midwifery training colleges should deepen ethical practice and attitudinal change the training of the midwives. This would create positive attitudes towards people of different background including the TBAs. Midwifery colleges should continue to widen the scope of the pre-service midwives practical to include visit to some of the trained TBAs. This would create friendly atmosphere between them, learn the practice and traditions of these TBAs and design strategies to communicate change to these TBAs tactfully.

\section{References}

Aborigo R. A, Allotey P, \& Reidpath D. D. The traditional healer in obstetric care: a persistent wasted opportunity in maternal health. Soc Sci Med. 2015;133(Supplement C):59-66. doi: 10.1016/j.socscimed.2015.03.046.

Adatara, P., Afaya, A., Baku, E. A., Salia, S. M., \& Asempah, A. (2018). Perspective of Traditional Birth Attendants on Their Experiences and Roles in Maternal Health Care in Rural Areas of Northern Ghana. International journal of reproductive medicine, 2018.

Abdul-Mumin, K.H (2016). Village midwives and their changing roles in Brunei Darussalam: a qualitative study. Women Birth. 2016;29(5):e73-e81. doi: 0.1016/j.wombi.2016.04.002.

Alligood, M. R. (2013). Nursing theory: Utilization \& application. Elsevier Health Sciences.

Asweto, C. O., Aluoch, J. R., Obonyo, C. O., \& Ouma, J. O. (2014). Maternal Autonomy, Distance to Health Care Facility and ANC Attendance Findings from Madiany Division of Siaya County, Kenya.

Aziato, L \& Omenyo, C. N (2018) Initiation of traditional birth attendants and their traditional and spiritual practices during pregnancy and childbirth in Ghana BMC Pregnancy Childbirth. doi: 10.1186/s12884-0181691-7

Bayou, Y. T. (2014). Maternal health care seeking behaviour and preferences for places to give birth in Addis 
Ababa, Ethiopia (Doctoral dissertation, University of South Africa).

Bonder, B., \& Martin, L. (2013). Culture in clinical care: Strategies for competence. Slack.

Bryce, J., Black, R. E., \& Victora, C. G. (2013). Millennium development goals 4 and 5: Progress and challenges. BMC medicine, 11(1), 1.

Byrne, A., \& Morgan, A. (2011). How the integration of traditional birth attendants with formal alth systems can increase skilled birth attendance. International Journal of Gynecology \& Obstetrics, 115(2), 127-134

Crissman, H. P., Engmann, C. E., Adanu, R. M., Nimako, D., Crespo, K., \& Moyer, C. A. (2013). Shifting norms: pregnant women's perspectives on skilled birth attendance and facility-based delivery in rural Ghana. African journal of reproductive health, 17(1), 15-26.

De Vos, A.S., Strydom, H., \& Fouche, C.B. (2009). Research at Grass roots. A Primer for the Caring Professions. Third Edition. Pretoria: Van Schaik.

Ebuehi, O. M., \& Akintujoye, I. A. (2012). Perception and utilization of traditional birth attendants by pregnant women attending primary health care clinics in a rural Local Government Area in Ogun State, Nigeria. Int $\mathbf{J}$ Womens Health, 4, 25-34

Dzakpasu, S., Soremekun, S., Manu, A., ten Asbroek, G., Tawiah, C., Hurt, L., ... \& Kirkwood, B. R. (2012). Impact of free delivery care on health facility delivery and insurance coverage in Ghana's Brong Ahafo Region. PloS one, 7(11), e49430.

Gillham, B. (2007). Research Interviewing. The range of techniques. Glasgow: Bell and Bain Ltd.

Kaingu, C. K., Oduma, J. A., \& Kanui, T. I. (2011). Practices of traditional birth attendants in Machakos District, Kenya. Journal of Ethnopharmacology, 137(1), 495-502.

Oshonwoh, F. E., Nwakwuo, G. C., \& Ekiyor, C. P. (2014). Traditional birth attendants and women's health practices: A case study of Patani in Southern Nigeria.

Journal of Public Health and Epidemiology, 6(8), 252-261.

Pfeiffer, C., \& Mwaipopo, R. (2013). Delivering at home or in a health facility? health-seeking behaviour of women and the role of traditional birth attendants in Tanzania. BMC pregnancy and childbirth, 13(1), 1.

Polit, D.F. \& Beck, C.T. (2004). Nursing Research: Principles and Methods. New York: Lippincott, Williams \& Wilkins.

Queku, I. C. (2016) Review of Compliance Measure in Empirical Accounting Literature: Perspective of International Financial Reporting Standards (IFRS). Research Journal of Finance and Accounting, 7(14), 5966

Riegel, B., Jaarsma, T., \& Strömberg, A. (2012). A middle-range theory of self-care of chronic illness. Advances in Nursing Science, 35(3), 194-204.

Sarmento, D. R. (2014). Traditional birth attendance (TBA) in a health system: what are the roles, benefits and challenges: a case study of incorporated TBA in Timor-Leste. Asia Pacific family medicine, 13(1), 1.

Sayegh, P., \& Knight, B. G. (2013). Cross-cultural differences in dementia: the Sociocultural Health Belief Model. International Psychogeriatrics, 25(04), 517-530.

Shaikh, B. T., Khan, S., Maab, A., \& Amjad, S. (2014). Emerging role of traditional birth attendants in mountainous terrain: a qualitative exploratory study from Chitral District, Pakistan. BMJ open, 4(11), $\mathrm{e} 006238$

Sumankuuro, J. (2014). Achieving MDGs: Breaking the silence on training of traditional midwifes in Developing Countries. Available at SSRN 2426849.

Titaley, C. R., Dibley, M. J., \& Roberts, C. L. (2012). Type of delivery attendant, place of delivery and risk of early neonatal mortality: analyses of the 1994-2007 Indonesia Demographic and Health Surveys. Health Policy and Planning, 27(5), 405-416.

Vyagusa, D. B., Mubyazi, G. M., \& Masatu, M. (2013). Involving traditional birth attendants emergency obstetric care in Tanzania: policy implications of a study of their knowledge and practices in Kigoma Rural District. International journal for equity in health, 12(1), 83.

Yawson, H. (2019) Relevance and challenges of traditional birth attendants' practices in the contemporary midwifery education and practice: Evidence from Kwesimintsim District in Ghana Journal of Health, Medicine and Nursing, 61(2019), 77-89 